\title{
SERIES INTRODUCTION
}

\section{Autoimmune diseases: are markers ready for prediction?}

\author{
Åke Lernmark \\ Department of Medicine, Robert H. Williams Laboratory, Box 357710 , \\ University of Washington, 1959 NE Pacific Street, Seattle, Washington 98195, USA. \\ Phone: (206) 543-5316; Fax: (206) 543-3169; E-mail: ake@u.washington.edu. \\ J. Clin. Invest. 108:1091-1096 (2001). DOI:10.1172/JCI200114234.
}

An increasing number of individuals throughout the world are affected by autoimmune disease, a large and diverse group of disorders that are categorized by tissue injury or pathology. In general, these diseases are associated with humoral or cell-mediated immune reactions against one or more of the body's own constituents, but it has been customary to divide autoimmune diseases into two categories - systemic, such as systemic lupus erythematosus, and organ-specific, such as autoimmune thyroiditis. The Perspective series beginning in this issue will address the ways in which autoantigen-specific responses arise, and the mechanisms by which they provoke cell type- and organ-specific pathologies. In addition, given our growing knowledge of etiologically significant autoantigens, one can envision that the occurrence of these diseases may soon be predictable in a clinical setting. How close are we, in fact, to taking this crucial step toward the management or prevention of autoimmune disorders?

\section{The identification of autoantigens}

Over the past four decades, the list of diseases associated with autoantibodies against tissues, cells, or specific autoantigens has grown enormously $(1,2)$. The classification of a disease as autoimmune has traditionally been based on the detection of autoantibodies that could be visualized reacting with an affected tissue or cell. Technological advances, in particular the development of microscopes that allow for more sensitive detection of cell surface-bound autoantibodies resulted in a proliferation of newly recognized autoimmune disorders. However, while such immunofluorescent images are often spectacular, they do not help to explain how the autoimmune response develops, nor even to identify the relevant autoantigen.

As was understood early on, the identification of individual autoantigens is key not only to uncovering the etiology and pathogenesis of an autoimmune disease, but also to improving the autoantibody assays used to diagnose or verify a disease. Improved biochemical and molecular methods have allowed a rapid dissection of autoantigens associated with specific autoimmune diseases. For analysis of peptide antigens, the most useful trick has been to use the patient's own autoantibodies as a biochemical tool in immunoprecipitation or immunoblotting experiments to identify the autoantigen, which can then be sequenced by standard techniques. Additional approaches have also been developed to characterize nonpeptide autoantigens. As can been seen in Table 1, there is now a smorgasbord of autoantigens that have been either cloned and sequenced or purified, many of which are commercially available as recombinant proteins and can be used for specific autoantibody assays. The molecular approach not only allows new and more specific assays to be established but sets the stage for structural analysis of epitopes by site-directed mutagenesis. Recombinant autoantigens facilitate studies of antibody isotype and subtype specificities and their possible association with autoimmune pathogenesis.

The availability of the autoantigen and its sequence has also made it possible to study autoantigen uptake and processing, the cell-surface presentation of epitopes on HLA class II molecules, and the role of B lymphocytes in autoantibody production. Recombinant autoantigens are also being produced in quantities sufficient to grow crystals for $x$-ray analysis of the structure, which should yield information crucial to uncovering $T$ and $B$ cell epitopes. Fortunately, alongside of this arduous process, it is possible to search the primary sequence of the autoantigen using several search algorithms based on the structural requirements for $\mathrm{T}$ cell receptor recognition of HLA-bound peptides. The availability of autoantigen has made it possible to isolate monoclonal antibodies from patients and to clone and sequence the heavy and light chain genes that encode the autoantibodies. Such analyses have revealed that there are many more replacements than silent mutations in the variable gene region and that replacement mutations have accumulated in the complementarity determining regions (CDRs). Since CDRs confer antigen-binding specificity, these studies therefore support the notion that the autoantigen is the driver of autoimmunity.

The availability of cloned autoantigens has not only changed the prospects of research into mechanisms of autoimmunity, but also improved the diagnostic sen- 
Table I

Recombinant or purified autoantigens recognized by autoantibodies associated with human autoimmune disorders

\section{Autoantigen}

A. Cell or organ-specific autoimmunity

Acetylcholine receptor

Actin

Adenine nucleotide translocator (ANT)

$\beta$-Adrenoreceptor

Aromatic L-amino acid decarboxylase

Asialoglycoprotein receptor

Bactericidal/permeability-increasing protein (Bpi)

Calcium-sensing receptor

Cholesterol side-chain cleavage enzyme (CYPIla)

Collagen type IV $\alpha_{3}$-chain

Cytochrome P450 2D6 (CYP2D6)

Desmin

Desmoglein 1

Desmoglein 3

F-actin

GM gangliosides

Glutamate decarboxylase (GAD65)

Glutamate receptor (GLUR)

$\mathrm{H} / \mathrm{K}$ ATPase

17- $\alpha$-Hydroxylase (CYP17)

21-Hydroxylase (CYP21)

IA-2 (ICA512)

Insulin

Insulin receptor

Intrinsic factor type 1

Leukocyte function-associated antigen (LFA-1)

Myelin-associated glycoprotein (MAG)

Myelin basic protein

Myelin oligodendrocyte glycoprotein (MOG)

Myosin

p-80-Coilin

Pyruvate dehydrogenase complex-E2 (PDC-E2)

Sodium iodide symporter (NIS)

SOX-10

Thyroid and eye muscle shared protein

Thyroglobulin

Thyroid peroxidase

Thyrotropin receptor

Tissue transglutaminase

Transcription coactivator p75

Tryptophan hydroxylase

Tyrosinase

Tyrosine hydroxylase

\section{B. Systemic autoimmunity}

$\mathrm{ACTH}$

Aminoacyl-tRNA histidyl synthetase

Aminoacyl-tRNA synthetase (several)

Cardiolipin

Carbonic anhydrase II

Collagen (multiple types)

Centromere-associated proteins

DNA-dependent nucleosome-stimulated ATPase

Fibrillarin

Fibronectin
Autoimmune disease

Myasthenia gravis

Chronic active hepatitis, primary biliary cirrhosis

Dilated cardiomyopathy, myocarditis

Dilated cardiomyopathy

Autoimmune polyendocrine syndrome type I (APS-I)

Autoimmune hepatitis

Cystic fibrosis vasculitides

Acquired hypoparathyroidism APS-I

Goodpasture syndrome

Autoimmune hepatitis

Crohn disease, coronary artery disease

Pemphigus foliaceus

Pemphigus vulgaris

Autoimmune hepatitis

Guillain-Barré syndrome

Type 1 diabetes, stiff man syndrome

Rasmussen encephalitis

Autoimmune gastritis

APS-I

Addison disease

Type 1 diabetes

Type 1 diabetes, insulin hypoglycemic syndrome (Hirata disease)

Type $B$ insulin resistance, acanthosis,

systemic lupus erythematosus (SLE)

Pernicious anemia

Treatment-resistant Lyme arthritis

Polyneuropathy

Multiple sclerosis, demyelinating diseases

Multiple sclerosis

Rheumatic fever

Atopic dermatitis

Primary biliary cirrhosis

Graves disease, autoimmune hypothyroidism Vitiligo

Thyroid associated ophthalmopathy

Autoimmune thyroiditis

Autoimmune Hashimoto thyroiditis

Graves disease

Coeliac disease

Atopic dermatitis

APS-I

Vitiligo, metastatic melanoma

APS-

ACTH deficiency

Myositis, dermatomyositis

Polymyositis, dermatomyositis

SLE

SLE, Sjögren syndrome, systemic sclerosis Rheumatoid arthritis (RA), SLE,

progressive systemic sclerosis

Systemic sclerosis

Dermatomyositis

Scleroderma

SLE, RA, morphea 


\section{Table 1 continued}

Autoantigen

Glucose-6-phosphate isomerase

$\beta 2$-Glycoprotein I ( $\beta 2$-GPI)

Golgin $(95,97,160,180)$

Heat shock protein

Hemidesmosomal protein 180

Histone H2A-H2B-DNA

IgE receptor

Keratin

Ku-DNA-protein kinase

Ku-nucleoprotein

La phosphoprotein (La 55-B)

Myeloperoxidase

Proteinase 3 (PR3)

RNA polymerase I-III (RNP)

Signal recognition protein (SRP54)

Topoisomerase-I (Scl-70)

Tubulin

Vimentin

C. Plasma protein and cytokine autoimmunity

C1 inhibitor

C1q

Cytokines (IL-1 $\alpha$, IL-1 $\beta$, IL-6, IL-10, LIF)

Factor II, factor V, factor VII, factor VIII, factor IX, factor X, factor XI, factor XII, thrombin, vWF Glycoprotein IIb/IIIg and Ib/IX

$\operatorname{IgA}$

Oxidized LDL (OxLDL)

D. Cancer and paraneoplastic autoimmunity

Amphiphysin

Cyclin B1

DNA topoisomerase II

Desmoplakin

Gephyrin

Hu proteins

Neuronal nicotinic acetylcholine receptor

p53

p62 (IGF-II mRNA-binding protein)

Recoverin

Ri protein

$\beta \mathrm{IV}$ spectrin

Synaptotagmin

Voltage-gated calcium channels

Yo protein

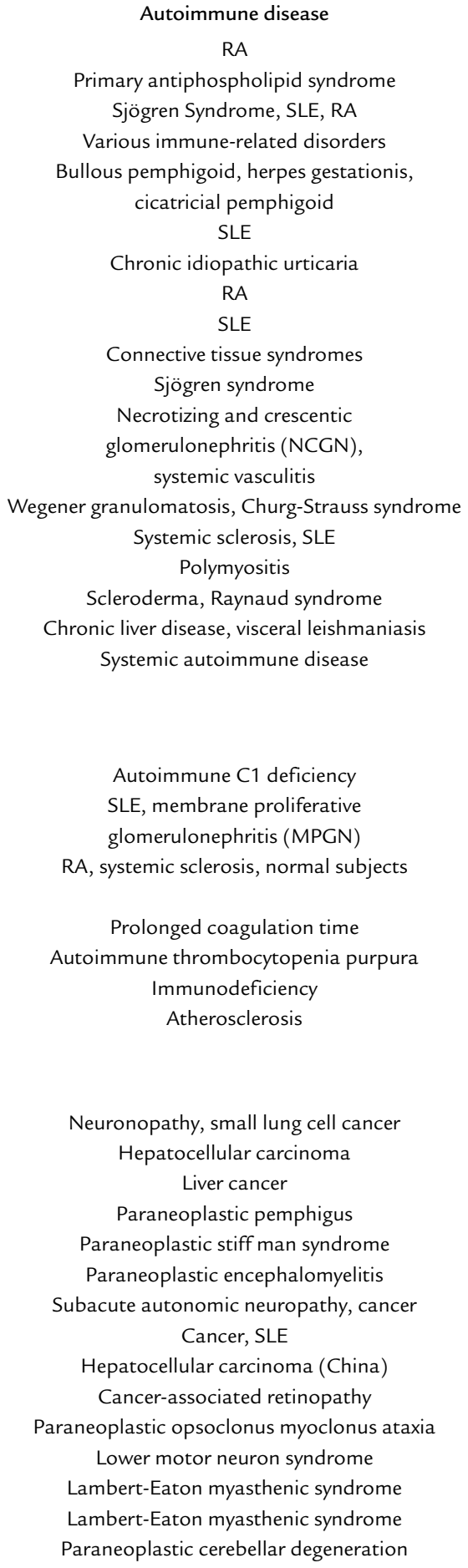

Neuronopathy, small lung cell cancer Hepatocellular carcinoma Liver cancer

Paraneoplastic pemphigus Paraneoplastic stiff man syndrome

Paraneoplastic encephalomyelitis

Subacute autonomic neuropathy, cancer Cancer, SLE

Hepatocellular carcinoma (China)

Cancer-associated retinopathy

Paraneoplastic opsoclonus myoclonus ataxia

Lower motor neuron syndrome

Lambert-Eaton myasthenic syndrome

Lambert-Eaton myasthenic syndrome

Paraneoplastic cerebellar degeneration 
sitivity and specificity of autoantigen-based autoantibody assays. In some instances, recombinant autoantigens have made it possible to standardize and quantify autoantibody assays (3) as well as to help develop reproducible $T$ cell tests (4). Standardization of autoantibody assays is critical to their use in the clinic to predict, diagnose, and treat this very diverse group of disorders. Since T cell-based tests have yet to be developed and standardized, it is likely that many of the estimated $5 \%$ of individuals in the US (5) with one or another autoimmune condition will benefit from the use of reliable autoantibody tests to predict disease.

\section{Pathogenesis of autoimmune disorders}

The list of autoantigens in Table 1 is not exhaustive but is meant to illustrate the precision by which autoantibodies to individual autoantigens serve as markers of major autoimmune diseases, as well as quite a few rare ones. This is to be expected, since the individual autoantigen most likely directs the autoimmune reaction to the site where the autoantigen is expressed. What is perhaps more surprising is that autoimmune attacks do not necessarily occur at all locations where the autoantigen is expressed. When the immune attack occurs, it is often executed by lymphocytes as well as autoantibodies, and many studies now indicate that prior views that autoimmunity is either cell-mediated or humoral have been an oversimplification (6). Regardless of the mechanisms, the precision of the immune reaction is remarkable. The autoimmune reaction may specifically ablate one cell type from an organ, such as the $\beta$ cells from the islets of Langerhans, without affecting the neighboring endocrine cells. The ablation of cells (and sometimes almost an entire organ, such as the thyroid) is a testimony to the efficiency by which the immune system eventually rids the host of an unwanted antigen.

The list of events that may turn an otherwise protective immune system into a vicious enemy from within can be made very long, and most autoimmune responses should at this point be regarded as entirely idiopathic. However, idiopathic autoimmunity often develops in subjects with a certain genetic propensity, most often linked or associated with HLA on chromosome 6 . The immune response to an autoantigen may also be initiated by cancer. Cancer-associated neoantigens are perhaps viewed as foreign by the immune system due to loss in immunological tolerance or an immune response that is able to overcome self-tolerance (see the article by Tan in this series). In other autoimmune disorders, the autoimmune disease is iatrogenic. Therapy with certain drugs may induce an autoimmune reaction. Even in these cases, however, HLA may still be critical to the risk of developing drug- or treatment-associated autoimmunity. Other etiologies are infectious diseases that may cause collateral autoimmune damage. Again, the autoimmune reaction is dependent on an individual's genetic propensity. The possibility that molecular mimicry (see the article by Wucherpfennig in this series) is a mechanism for human autoimmune disease has been discussed for thirty years (7). The interplay between the environment and genetics is also exempli- fied by a condition such as celiac disease (CD), which is caused by intolerance to gluten, a major constituent of wheat. After diagnosis, removal of gluten from the diet is the only current treatment for the disease (see the article by Papadopoulos et al. in this series).

The immune system may be fooled into reacting with autoantigens following an initial reaction to infectious agents. The possible mechanisms by which infectious agents activate autoreactive $\mathrm{T}$ and $\mathrm{B}$ lymphocytes are discussed by Wucherpfennig in this series. One possibility that has been entertained by many authors is induction of immune response by molecular mimicry, i.e., a structural similarity between microbial and selfantigens. Several human diseases involve superantigens, molecules that can activate $\mathrm{T}$ cells directly through interactions with the variable domain of the $T$ cell receptor $\beta$ chain. In experimental animals, there is considerable evidence that superantigens can initiate autoimmunity, but their roles in human autoimmunity are less clear, and other mechanisms, such as release of autoantigens following viral lysis, activation of lymphocytes by virus, or bystander activation, may also contribute to these disease processes. In humans, there are several examples of autoimmune disease with an acute onset that follow infection. Rheumatic fever is but one example. The development of Guillain-Barré syndrome after infection with Campylobacter jejunum is another. Two rheumatological diseases, reactive arthritis and Lyme arthritis, have strong links to defined bacterial agents. Importantly, some of these diseases show the same HLA association as rheumatoid arthritis. A deeper understanding of the relationship between infection and autoimmunity may allow the prevention of autoimmune sequelae in some of these diseases.

\section{Organ-specific autoimmunity}

This series will update our understanding of a select few organ-specific autoimmune diseases, including thyroid autoimmunity, type 1 diabetes, and CD (see the articles by Rapaport and Maclachlan, Notkins and Lernmark, and Papadopoulos et al. in this series). In all three diseases, major breakthroughs in the identification of the relevant autoantigens have allowed the development of autoantigen-specific autoantibody assays, which can be used for disease classification as well as prediction. Prediction may also be relevant to tumorigenesis, since autoantibodies that report the presence of a tumor can now be specifically detected in addition to autoantibodies in paraneoplastic disease that indirectly flag the presence of a tumor. The concept that autoimmunity can emerge from aberrant cellular growth has long been debated. A better understanding of the autoantigens involved and the development of autoantigen-specific antibody or T cell assays may help to pave the way for future clinical applications. Similarly, numerous questions remain to be answered regarding the immune responses to infection and the resulting induction of autoimmunity. For instance, would measurements of autoantibodies be useful in predicting whether an infection has placed a patient at risk of autoimmune disease? 
Hashimoto thyroiditis was reported to be an autoimmune disease more than forty years ago and, together with Graves disease, has served as a model disease for the dissecting of disease pathogenesis. Hashimoto thyroiditis and Graves disease are also the most common organ-specific autoimmune diseases affecting humans, as discussed by Rapaport and Maclachlan in this series. Ironically, due to the availability of a relatively effective replacement therapy and the diseases' onset at an older age and general lack of life-threatening complications, there has been little impetus to find a cure or develop preventative measures for these conditions. The study of Hashimoto thyroiditis and Graves disease has, however, been markedly accelerated by the identification, molecular cloning, and expression of dominant and specific autoantigens, namely thyroid peroxidase and thyrotropin receptor. While the pathogenetic role of autoantibodies against thyroid peroxidase is much debated, it now seems clear that the thyrotropin receptor antibodies can mimic the action of the thyrotropin and directly stimulate the thyroid to cause hyperthyroidism. Impressive progress is being made to identify $\mathrm{B}$ and $\mathrm{T}$ cell epitopes as well as the structural constraints of the autoantigens by ongoing crystallization efforts, but thyroid antigen uptake, processing, and presentation and the possible role of $\mathrm{B}$ cells in presenting autoantigen to $T$ cells remain poorly understood. Rapaport and Maclachlan argue that the effort to identify non-HLA-contributing genes will be of little help, since even when the process of antithyroid reactivity is established, the actual mechanism by which the thyroid cells are damaged will still need to be clarified. The bottom line for these authors is that an understanding of the antigen-specific immune responses holds the key to future progress. Autoantibody assays will need to be developed and standardized using internationally recognized reference sera if they are to be used to predict thyroid disease. Unfortunately, suitable treatments for individuals who test positive for these autoantibodies are by no means obvious.

Similar unresolved issues in type 1 diabetes are also discussed by Notkins and Lernmark in this series. In type 1 diabetes it has been possible to identify three autoantigens: the smaller isoform of glutamate decarboxylase, GAD65; IA-2, also known as ICA512, an unusual transmembrane protein tyrosine phosphatase; and insulin. Much effort has been devoted to the development of standardized antibody assays in international serum-exchange workshops (8). This effort, initiated by the Immunology of Diabetes Workshops in 1985 and now continued through the effort of the Immunology of Diabetes Society in a partnership with the Center of Disease Control and Prevention, has resulted in a reference standard for both islet cell antibodies (ICAs) and GAD65 autoantibodies (3). The presence of autoantibodies to all three antigens has emerged as a strong predictor of disease. Several screening studies of newborn babies, school children, and adults, focusing in particular on first-degree relatives of affected individuals, have been carried out to test whether it is possible to reduce the risk for type 1 diabetes. In particular, nondiabetic but autoantibody-pos- itive subjects have been administered parenteral, oral, or nasal insulin, nicotinamide, or baby formula free of cow's-milk proteins (9).

Screening studies in newborns should help identify environmental factors that can trigger type 1 diabetes. In addition, case reports going back 100 years have shown a relationship between viral infections and type 1 diabetes, but the search for viral antigens has been deemphasized because already at clinical onset type 1 diabetes shows signs of a chronic autoimmune disease. While it is recognized that type 1 diabetes development is strongly associated with T cells infiltrating the islets of Langerhans, a precise, reproducible, and standardized $\mathrm{T}$ cell assay has yet to be established. Nonetheless, the extensive work to standardize the autoantibody assays has to a very large degree made type 1 diabetes a predictable disease. The challenging next step will be to develop either refined autoantibody assays or tests of cellular immunity that show higher diagnostic sensitivity and specificity and better predictive ability than currently available autoantibody assays.

The situation in CD, as discussed by Papadopoulos et al., is somewhat different. As with thyroid disease but not type 1 diabetes, it is possible to verify the diagnosis by a biopsy of the affected tissue. In active CD, the intestinal villus is completely flattened by inflammation, but the gut regains its normal structure and absorptive function once a gluten-free diet is introduced. CD is strongly associated with certain HLA haplotypes, and since $\mathrm{T}$ cells in the intestinal biopsies are available for study, it has been possible for this, more than for any other, autoimmune disease to determine the role of HLA in presenting antigen-gluten to T cells. The recent demonstration that tissue transglutaminase is a CD autoantigen has generated increased interest in developing specific, sensitive radioligand assays to replace the diagnostic test that is now widely used, an immunofluorescence assay for anti-endomysial antibodies in a patient's serum. It has been hypothesized that tissue transglutaminase modifies gluten peptides, rendering them antigenic, and that, during the course of this interaction, the enzyme itself becomes recognized as antigen. Modeling studies of HLA class II molecules with gluten peptides has provided novel insights into possible mechanisms of the structural constraints of antigen presentation. Papadopoulos et al. focus on a different question, however: Why it is only a select few of the many subjects with high-risk HLA genotypes that eventually are affected by CD? These authors (unlike Rapaport and Maclachlan in their discussion of thyroid disease) favor the search for non-HLA genetic factors, but they also consider whether we may have overlooked other, nondietary environmental factors that increase the risk of CD.

\section{Cancer and autoimmunity}

While thyroiditis, type 1 diabetes, and CD have strong environmental components, this is not the case in cancer-associated autoimmunity. As discussed by Tan, the immune reaction provoked by tumors is directed against antigens present in the tumor cell. Whereas autoantigens are typically normal, nonmutated mole- 
cules, in cancer immunity, one pathway may involve genetic mutation of the autoantigen recognized by patient autoantibodies, as is the case for the p53 tumor suppressor protein. Although the clinical utility of $\mathrm{p} 53$ autoantibodies to either predict or monitor cancer treatment is far from certain, preliminary data suggest that the immune system can in some instances sense the existence of structurally altered p53 prior to the appearance of clinical symptoms. Interestingly, some of the autoantigens associated with tumors are seen in healthy organisms only during fetal development. Such onco-fetal epitopes may therefore flag the immune system when they reemerge later in life. This area of research has important clinical implications, and the rate at which autoantigens have been identified, cloned, sequenced, and expressed suggests that these molecules hold great promise as tumor risk reporters. To date, however, there is a paucity of data on the HLA association - an important gap to fill.

Symptoms of autoimmune disease may also serve as tumor reporters, much as complaints of referred pain can alert a physician to the growth of a tumor at a distant site. Tumors of the lung, breast, ovary, and testis, which provoke autoimmune responses in situ, are likewise associated with a host of neurological abnormalities, collectively referred to as paraneoplastic disorder syndrome. Fortunately, neurological symptoms in these disorders typically disappear after the tumor has been removed. Again, the autoantigens recognized have been identified in several spectacular discoveries (Table 1).

\section{Autoantibodies and disease prediction}

Since individual autoantibodies can serve as tumor reporters for monitoring treatment, it seems likely that a panel of tumor-associated antigens would have some utility in predicting cancer. Likewise, specific autoantibodies might well be used as markers of an ensuing autoimmune disease - an approach considered by Leslie et al. in the final article in this series. These authors point out that the detection of autoantibodies has proved remarkably valuable in the clinical management of certain diseases but that autoantibodies remain underutilized as predictive markers. Diseases with a long preclinical period are of particular interest to target for prediction. For example, individuals with 21-hydroxylase antibodies are likely to eventually devel- op Addison disease, and such people could be diagnosed and treated well before the potentially fatal clinical symptoms appear. Autoantibody assays can also be useful to classify disease. Thus, there are several causes of atrophic gastritis and vitamin B12 deficiency, but the presence of autoantibodies to H/K ATPase and intrinsic factor indicates that the disease is autoimmune gastritis. Furthermore, given sufficient sensitivity, specificity, and positive predictive value, autoantibody assays may even allow certain diseases to be averted altogether. These features will have to be validated using reliable assays and prospective studies, but they are crucial to avoid unnecessary overtreatment, i.e., treatment of subjects who would not have developed the disease if left alone. Diseases currently amenable to prediction include type 1 diabetes, thyroiditis, $\mathrm{CD}$, Addison disease, pernicious anemia, and rheumatic diseases.

Although many issues remain unresolved, Leslie et al. conclude that screening populations for susceptibility to certain autoimmune diseases is now feasible. New high throughput methods will permit low-cost rapid screening of dozens of autoantibodies, and such screening may become a routine part of a medical examination. The benefit and cost saving of such practice will be tremendous once preventive measures and safe therapies become available.

1. Rose, N.R., and Mackay, I.R. 1998. Autoimmune diseases. 3rd edition. Academic Press Inc. San Diego, California, USA. 895 pp.

2. Peter, J.B., and Shoenfeld. Y. 1996. Autoantibodies. Elsevier Science B.V. Amsterdam, The Netherlands. 880 pp.

3. Mire-Sluis, A.R., Gaines Das, R., and Lernmark, Å. 2000. The World Health Organization International Collaborative Study for islet cell antibodies. Diabetologia. 43:1282-1292.

4. Peakman, M., et al. 2001. Characterization of preparations of GAD65, proinsulin, and the islet tyrosine phosphatase IA-2 for use in detection of autoreactive T-cells in type 1 diabetes: report of phase II of the Second International Immunology of Diabetes Society Workshop for Standardization of T-cell assays in type 1 diabetes. Diabetes. 50:1749-1754.

5. Jacobson, D.L., Gange, S.J., Rose, N.R., and Graham, N.M. 1997. Epidemiology and estimated population burden of selected autoimmune diseases in the United States. Clin. Immunol. Immunopathol. 84:223-243.

6. Davidson, A., and Diamond, B. 2001. Autoimmune diseases. N. Engl. J. Med. 345:340-350.

7. Albert, L.J., and Inman, R.D. 1999. Molecular mimicry and autoimmunity. N. Engl. J. Med. 341:2068-2074.

8. Verge, C.F., et al. 1998. Combined use of autoantibodies (IA-2 autoantibody, GAD autoantibody, insulin autoantibody, cytoplasmic islet cell antibodies) in type 1 diabetes: Combinatorial Islet Autoantibody Workshop. Diabetes. 47:1857-1866.

9. Rabinovitch, A., and Skyler, J.S. 1998. Prevention of type 1 diabetes. Med. Clin. North Am. 82:739-755. 